\title{
Characteristics and Outcomes of Ventricular Tachycardia and Premature Ventricular Contractions Ablation in Patients with Prior Mitral Valve Surgery
}

Fouad Khalil ${ }^{1}$, Takumi Toya ${ }^{1}$, Malini Madhavan ${ }^{1}$, Mohammed Badawy ${ }^{1}$, Suraj Kapa ${ }^{1}$, Siva Mulpuru $^{1}$, Konstantinos Siontis ${ }^{1}$, Christopher DeSimone ${ }^{1}$, Abhishek Deshmukh ${ }^{1}$, Young-mei Cha ${ }^{1}$, Paul Friedman ${ }^{1}$, Thomas Munger ${ }^{2}$, Samuel Asirvatham ${ }^{1}$, and Ammar $\mathrm{Killu}^{1}$

${ }^{1}$ Mayo Clinic

${ }^{2}$ Mayo Clinic West $16 \mathrm{~A}$

July 2, 2021

\begin{abstract}
Background: Data regarding ventricular tachycardia (VT) or premature ventricular complex (PVC) ablation following MVS is limited.) CA can be challenging given perivalvular substrate in the setting of mitral annuloplasty or prosthetic valves. Objective: To investigate the characteristics, safety, and outcomes of radiofrequency catheter ablation (CA) in patients with prior mitral valve surgery (MVS) and ventricular arrhythmias (VA). Methods: We identified consecutive patients with prior MVS who underwent CA for VT or PVC between January 2013- December 2018. We investigated the mechanism of arrhythmia, ablation approach, peri-operative complications, and outcomes. Results: In our cohort of 31 patients $(77 \%$ men, mean age $62.3 \pm 10.8$ years, left ventricular ejection fraction $39.2 \pm 13.9 \%$ ) with prior MVS underwent CA (16 VT; 15 PVC). Access to the left ventricle was via transseptal approach in 17 patients, and a retrograde aortic approach was used in 13 patients. A combined transseptal and retrograde aortic approach was used in one patient, and a percutaneous epicardial approach was combined with trans-septal approach in 1patient. Heterogenous scar regions were present in $94 \%$ of VT patients and scar-related reentry was the dominant mechanism of VT. Clinical VA substrates involved the peri-mitral area in 6 patients with VT and 5 patients with PVC ablation. No procedure-related complications were reported. The overall recurrence-free rate at 1-year was $72.2 \%$; $67 \%$ in the VT group and $78 \%$ in the PVC group. No arrhythmia-related death was documented on long-term follow-up. Conclusion: CA of VAs can be performed safely and effectively in patients with MVS
\end{abstract}

\section{Characteristics and Outcomes of Ventricular Tachycardia and Premature Ventricular Contrac- tions Ablation in Patients with Prior Mitral Valve Surgery}

Short Title : Ventricular Arrhythmia in Patients with prior Mitral Valve Surgery

Fouad Khalil, M.B.,B.Ch. ${ }^{1}$; Takumi Toya, M.D. ${ }^{1}$; Malini Madhavan, M.B.B.S. ${ }^{1}$, Mohamed Badawy, M.B.,B.Ch ${ }^{1}$; Suraj Kapa M.D. ${ }^{1}$, Siva K. Mulpuru M.D., FHRS. ${ }^{1}$, Konstantinos C. Siontis, MD ${ }^{1}$; Christopher V. DeSimone, MD PhD ${ }^{1}$ : Abhishek J Deshmukh M.B.B.S. ${ }^{1}$, Yong-Mei Cha, $\mathrm{MD}^{1}$; Paul A. Friedman, M.D. ${ }^{1}$, Thomas Munger, M.D. ${ }^{1}$, Samuel J. Asirvatham, M.D., FHRS. ${ }^{1}$, Ammar M. Killu ${ }^{1}$, MBBS.

1 Department of Cardiovascular Medicine, Mayo Clinic, Rochester, MN.

\section{Corresponding author:}

Ammar M. Killu, MBBS 
200 First Street SW

Rochester, MN 55905

Tel: $507-538-6325$

Fax: 507-255-2550

Email: killu.ammar@mayo.edu

Disclosures : None

Funding : None

Word count: 3746 (excluding references)

\begin{abstract}
Background: Data regarding ventricular tachycardia ( VT) or premature ventricular complex (PVC) ablation following MVS is limited.) CA can be challenging given perivalvular substrate in the setting of mitral annuloplasty or prosthetic valves.
\end{abstract}

Objective: To investigate the characteristics, safety, and outcomes of radiofrequency catheter ablation (CA) in patients with prior mitral valve surgery (MVS) and ventricular arrhythmias (VA).

Methods: We identified consecutive patients with prior MVS who underwent CA for VT or PVC between January 2013- December 2018. We investigated the mechanism of arrhythmia, ablation approach, perioperative complications, and outcomes.

Results: In our cohort of 31 patients ( $77 \%$ men, mean age $62.3 \pm 10.8$ years, left ventricular ejection fraction $39.2 \pm 13.9 \%$ ) with prior MVS underwent CA (16 VT; 15 PVC). Access to the left ventricle was via transseptal approach in 17 patients, and a retrograde aortic approach was used in 13 patients. A combined transseptal and retrograde aortic approach was used in one patient, and a percutaneous epicardial approach was combined with trans-septal approach in 1patient. Heterogenous scar regions were present in $94 \%$ of VT patients and scar-related reentry was the dominant mechanism of VT. Clinical VA substrates involved the peri-mitral area in 6 patients with VT and 5 patients with PVC ablation.

No procedure-related complications were reported. The overall recurrence-free rate at 1-year was $72.2 \% ; 67 \%$ in the VT group and $78 \%$ in the PVC group. No arrhythmia-related death was documented on long-term follow-up.

Conclusion : CA of VAs can be performed safely and effectively in patients with MVS

Key Words : ventricular tachycardia, premature ventricular complex, mitral valve, prosthesis

\title{
Introduction
}

Ventricular arrhythmia (VA) is an important cause of morbidity and mortality in patients with mitral valve disease. Several studies have illustrated the importance of VA as a predictor of sudden cardiac death in patients with mitral valve disease. ${ }^{1-5}$ Though mitral valve surgery (MVS) has shown significant improvement in heart failure symptoms and mortality, surgical intervention does not uniformly eliminate the risk of VA. ${ }^{6-9}$ Furthermore, valve surgery itself could be a contributing factor for VA occurrence either early following surgery or years later. ${ }^{10}$ The mechanism of VA in this population includes bundle branch reentry (BBR), enhanced automaticity, and scar-related reentry. The proximity of the His bundle to the mitral annulus makes BBR an important mechanism especially when VA presents early following $\mathrm{MVS}^{11}$, whereas scarrelated reentry tends to present later. Data about the characteristics and outcomes of VA in patients with prior MVS remains scant. ${ }^{10,12-16}$ Previous studies have investigated VA ablation in patients with cardiac or valve surgery in general, but not specifically in those with MVS. ${ }^{10}{ }^{14}$ Thus, MVS patients represented a minor category in prior reports. Furthermore, prior studies focused on early postoperative VA and only a few studies addressed late-onset VA. 
We report on a cohort of 31 patients with a prior history of MVS who were treated with CA for ventricular tachycardia (VT) or premature ventricular contractions (PVC). We describe the characteristics of arrhythmia as well as feasibility, safety and outcomes of catheter ablation procedures in this patient population.

\section{Methods}

\section{Patient cohort}

Following institutional review board approval (IRB), we identified all patients with prior MVS (replacement or repair) who developed ventricular arrhythmia (VA), defined as occurrence of VT or PVC who then underwent radiofrequency CA for this between January 2013 and December 2018. A total of 31 patients with prior history of MVS who underwent ablation for VT (16 patients) or PVC (15 patients) were identified. Patients with congenital heart disease were excluded. The Mayo Clinic comprehensive clinical record system was used to abstract data.

\section{Electrophysiology Study and Ablation}

Patients were taken to the electrophysiology laboratory in the fasting, postabsorptive state. The level of anesthesia was determined by the performing electrophysiologist. Intravenous prophylactic antibiotics were administered. Following vascular access, intravenous heparin was administered to achieve activated clotting time (ACT) of 300-350 seconds. An arterial line was placed for continuous blood pressure monitoring. A 5 French (Fr) quadripolar catheter was placed in the right ventricular apex and a $7 \mathrm{Fr}$ decapolar catheter was placed in the coronary sinus. Intracardiac echocardiography (ICE, Acuson, Siemens, Germany) was used as standard to assist mapping/ ablation and assess for complications. Left ventricular (LV) access was obtained by anterograde trans-septal or retrograde trans-aortic approach. 3-dimensional electroanatomical mapping (CARTO, Biosense Webster, Diamond Bar, CA) was used in all patients. Mapping was performed in point-by-

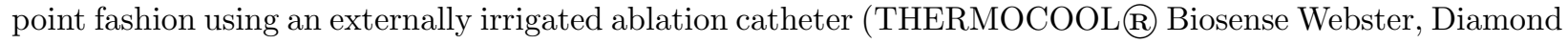
Bar, CA). For VTs, programmed ventricular stimulation and burst pacing were utilized for induction. For PVCs, burst pacing was favored if PVCs were scarce. Isoproterenol was utilized as needed for both. Standard mapping techniques were used to identify ablation targets. ${ }^{17}$ In the case of PVCs, activation and pace mapping was predominantly utilized. In VT cases, entrainment and activation mapping were used if the arrhythmia was hemodynamically tolerated. With unstable VT, pace and substrate mapping were favored. Scar-related reentry was defined as the VT mechanism when VT was inducible with programmed stimulation and when entrainment could be demonstrated. VT was considered involving the perimitral area in cases where abnormal electrograms and critical sites responsible for VT were identified within $2 \mathrm{~cm}$ of the valve annulus. Ablation energy was typically started at $30 \mathrm{~W}$ and up-titrated as necessary, up to a maximum limit of 50 W.

\section{Definitions}

The VT or PVC targeted during ablation was deemed as the "clinical" arrhythmia if it resembled the arrhythmia noted on preprocedural monitoring (ECG, ambulatory heart rhythm monitoring, ICD electrograms analysis). Procedural outcomes were defined as follows:

Procedural success was defined as no inducibility of VT or PVC following ablation

Procedural failure was characterized by continued presence of the clinical arrhythmia following ablation.

Postprocedural outcomes were defined as follows:

Clinical success was determined by no recurrence with the same/ fewer antiarrhythmic drugs (AAD) used in the case of VT and by $80 \%$ reduction in burden or improved symptoms with same/ less AAD use in the case of PVC ${ }^{18}$.

Clinical failure was determined by the recurrence of arrhythmia (less than $80 \%$ burden reduction in the case of PVC) or recurrent symptoms attributable to the arrhythmia, ICD therapies, or the need for a repeat ablation procedure. 


\section{Clinical follow-up}

Following hospital discharge, patients were followed up via telephone call at 30 days in addition to a followup office visit at 3 months, 12 months and annually thereafter. Arrhythmia recurrence was determined by history, clinical symptoms, non-invasive monitoring devices and device interrogation, when possible.

\section{Statistical analysis}

Continuous variables distributed normally were expressed as the mean \pm standard deviation, and those with a skewed distribution were expressed as the median (interquartile range [IQR]). Categorical variables were expressed as frequency (percentage). For between-group comparisons, unpaired t-test was used for normally distributed variables, Mann-Whitney $U$ test for non-normally distributed variables, and $\chi^{2}$ test (or Fisher exact test) for categorical variables. Kaplan-Meier methods were used to estimate survival or recurrencefree survival rates. The difference between patients with coronary artery diseases (CAD) and those without CAD was analyzed using the log-rank test. For all tests, a 2-tailed P-value $<0.05$ was considered statistically significant. All statistical analyses were performed using JMP Pro Software 14.3.0 (SAS Institute, Inc., Cary, North Carolina, USA) and GraphPad Prism 8.3.0 (GraphPad Software, La Jolla, California, USA).

\section{Results}

Baseline characteristics are summarized in Table 1. Between 2014 and 2018, 31 patients with a prior history of MVS (repair or replacement) underwent RFA for VT (16 patients) or PVC (15 patients). Seven (23\%) patients had undergone mitral valve replacement (3 patients had mechanical valves) and $24(77 \%)$ patients had prior mitral valve repair (23 with partial annuloplasty ring, 1 with complete annuloplasty ring). The majority of the patients were Caucasian (90\%) and male $(77 \%)$ with a mean age of $62.3 \pm 10.8$ years. The mean left ventricular ejection fraction (LVEF) was $39.2 \pm 13.9 \%$. Obstructive sleep apnea (OSA, 43\%), chronic kidney disease (CKD, 35\%), and hypertension (HTN, 33\%) were the most common comorbidities in our cohort. Seven patients had prior aortic valve surgery (3 of which were at the same time of MVS). All patients were receiving oral anticoagulation prior to the procedure. Four patients with mitral repair were using direct-acting oral anticoagulants (DOAC) while the rest of the cohort was on warfarin. Nine patients had mitral valve prolapse prior to MVS. There were no cases of mitral annular disjunction. Ten patients $(32 \%)$ had a history of known CAD - 5 patients had prior CABG, 3 patients had prior PCI and 2 patients had both CABG and PCI. Seven patients (23\%) had a history of prior ablation of VA at an outside institution.

\section{Electrophysiology study and ablation}

Procedural characteristics are summarized in Table 2. LV access was obtained through antegrade transseptal approach in 17 patients, retro-aortic approach in 13 patients and both antegrade and retrograde in one patient. In 1 patient, a percutaneous pericardial approach was combined with the antegrade trans-septal approach.

\section{Characteristics of the VT group (Table 3)}

The median duration between valve surgery and VT onset was 4.0 (IQR 0.3, 12.1) years while the median duration between MVS and VT ablation was 5.0 (IQR 1.0, 11.0) years. Twelve patients in this group had MV repair while 4 patients had MV replacement (of which 1 had a mechanical valve). Seventy percent of the VT group had ICD in situ prior to ablation, the rest had ICD implantation after the ablation. Of the 16 patients in this group, 13 patients (81\%) had failed [?]1 AAD, 11 (85\%) of whom had failed a Class III AAD prior to ablation. The median LVEF was 28 (IQR 20, 36). The mean LVEDD was66 +-9 mm. The mean RVSP was42 +-16 mmHg. General anesthesia was used in 7 (40\%) patients. Thirty-seven VTs were induced with a median of 2 (IQR 1,3). Seventy percent of induced VTs had RBBB morphology with superior axis being the predominant axis. Thirty percent of induced VTs had LBBB morphology where the origin of VT was found to be RV, interventricular septal, or mitral isthmus. The median VT cycle length was 378 (IQR $298,477)$ ms. Scar-related reentry was the major mechanism of induced VTs. Only one patient had BBR VT which occurred following a complicated aortic valve replacement surgery. 
Substrate mapping demonstrated heterogenous scar that involved the perimitral valve area in $8(50 \%)$ patients. Despite that, the site of successful ablation included the perimitral region only in 6 patients.

Procedural success was achieved in $94 \%$ of the VT group. Only one patient had procedural failure. This patient had an EF of $20 \%$ with a history of NICM and prior VT ablation. During ablation, induced VTs were associated with hemodynamic instability requiring multiple defibrillations and the use of a percutaneous left ventricular assist device. The critical isthmus was felt to be epicardial.

The median total procedure time for the VT group was 298.5 (IQR 414.8, 226.5) minutes and median fluoroscopy time was 35.65 (IQR 52.0, 26.1) minutes. The median energy delivery time was 64.0 (IQR 95.3, 34.7) minutes. Following the procedure, all patients in the VT group were continued on their preprocedural AAD regimen.

\section{Characteristics of PVC group (Table 4)}

The median duration between valve surgery and PVC onset was 3.4 (IQR 0.6, 8.0) years while the median duration between MVS and PVC ablation was 4.0 (IQR 1.0, 10.0) years. Twelve patients in this group had MV repair while 3 patients had MV replacement (of which 2 had mechanical valve). The median LVEF is 48(IQR 43, 53). Thirty-three percent of the PVC group had ICD in situ prior to ablation. The mean LVIDd was $55+-9 \mathrm{~mm}$. The mean RVSP was $27+-8 \mathrm{mmHg}$. Thirteen patients $(87 \%)$ in the PVC group had RBBB morphology with an inferiorly directed axis in 9 cases and a superior axis in 4 cases. Eleven (73\%) out of the 15 patients in the PVC group had mapping of the perimitral area. Of these, signal abnormalities consistent with scar in the perimitral area were noted in $5(45 \%)$ patients. In 9 patients with PVCs, the successful site of ablation was unrelated to the perimitral area. Rather, $20 \%$ of patients had successful ablation from the posterior papillary muscle; all of them had prior history of mitral valve repair with posterior annuloplasty ring and commissuroplasty. The remainder of PVCs were mainly of outflow tract origin. One patient had PVC originating from the septal RVOT while in another, PVC originated from the lateral RV inflow. The local ventricular activation time recorded at successful ablation targets that preceded the QRS onset was 34 +-8 millisecond. Procedural success was achieved in $93 \%$ of the PVC group. Only one patient had procedural failure. In this patient, the clinical PVC was mapped to the epicardial LV "summit" region where RF ablation was limited due to proximity to the left anterior descending coronary artery. This patient was discharged on amiodarone.

The median total procedure time for the PVC group was 255 (IQR 318.25, 191) minutes and median fluoroscopy time was 28 (IQR 46, 12.3) minutes. The median energy delivery time was 20.9 (IQR 30.6, 9.5) minutes.

\section{VA ablation-related complications}

There were no major complications in either group. Intracardiac echocardiography at the end of the procedure and pre-dismissal TTE revealed no pericardial effusion or change in the mitral valve function in both groups. There were no cases of catheter entrapment, stroke/ TIA, or infective endocarditis in both groups.

Follow-up after VA ablation

Survival and recurrence-free survival are shown in Figure 1Aand 1B, respectively. The overall recurrencefree rate at 1-year for the whole cohort was $72 \% ; 67 \%$ in the VT group vs. $78 \%$ in the PVC group.

During a median follow-up of 478 days (IQR 121, 894) after VA ablation, 5 patients died in the VT group, whereas no patients in the PVC group died. There were no procedure or arrhythmia-related deaths. Two patients died from progressive heart failure, one patient died from respiratory failure and the cause of death was unknown in 2 patients.

There was no significant difference in survival after VA ablation between patients with a history of CAD or without history of $\mathrm{CAD}$ ( $\log \operatorname{rank} \mathrm{P}=0.82)$ (Figure 2A ); however, there was a trend that patients without history of CAD had lower recurrence-free survival than those with CAD ( $\log$ rank $\mathrm{P}=0.06)$ (Figure 2B ). 


\section{Discussion}

In this study of catheter ablation for symptomatic VT and PVC in patients with prior MVS, we demonstrated the following: 1. Catheter ablation is an effective treatment modality with reasonable long term outcomes, 2. The clinical arrhythmia, be it VT or PVC, was frequently not related to the perimitral region, 3. Most patients presented late after MVS with an average time latency of 4.0 (IQR 0.3, 12.1) and 3.4 (QR 0.6, 8.0) years for VT and PVCs onset, respectively and 4. Catheter ablation is safe in this patient population, with no instances of catheter entrapment or mitral valve malfunction.

Following cardiac valve surgery, atrial arrhythmias are much more frequently encountered than VAs. ${ }^{19-21}$ As such, characteristics of VA and the associated ablation in this population have not been well delineated. Our methods did not permit us to establish the incidence of VA following MVS. However, there is some limited data with respect to acute postoperative VA incidence. A large study for post-cardiac surgery outcomes included 813 patients with valve surgery. Of these, only $6(0.74 \%)$ patients developed sustained VT post valve surgery. ${ }^{22}$. Though this study included 749 patients with MVS, the incidence of VT was reported after valve surgery in general. Another study reported that the incidence of VT was $8 \%$ in patients with mitral valve replacement compared to $21 \%$ with aortic valve replacement. ${ }^{23}$ Though these studies evaluated acute incidence, VA can present years following surgical intervention, akin to postoperative atrial flutter and post myocardial infarction ventricular arrhythmias. ${ }^{10}$ Indeed, variation in VA onset following cardiac surgery might be related to the difference in VA mechanisms, for example early-onset being associated with neurohormonal imbalance, automaticity with later onset being more likely to be associated with reentry.

\section{Mechanism and onset}

The mechanism of VA in patients with MVS can include increased automaticity, BBR VT, and scar-related reentry. In our analysis, scar-related reentry was the major mechanism of arrhythmia. Increased automaticity and BBR were documented mostly with early-onset VT. The proximity of the His bundle to the mitral valve annulus makes it vulnerable to any pathological process or surgery involving the valve. ${ }^{24,25}$ Furthermore, hemorrhagic trauma to the His bundle and proximal bundle branches has been documented after MVS. ${ }^{25}$ These factors could facilitate BBR VT in the setting of elevated catecholamines and autonomic imbalance postoperatively. While VT has been well delineated in this patient cohort, less is known about non-BBR VT in these patients. ${ }^{10,12-16}$ Unlike BBR VT, scar-related reentry tends to be implicated in late-onset VA. A study of 29 patients with VT after valve surgery found that the BBR VT was responsible for VT in 8 (73\%) of the 11 patients who presented within 30 days after surgery. ${ }^{11}$ Conversely, of 18 patients presenting with VT more than 30 days after their valve surgery, 17 had "myocardial" VT with only 1 having BBR VT. Though the mechanism of VT was not determined in these 17 patients, it is assumed that scar-related reentry is most likely given that $75 \%$ of these patients had CAD and reduced EF.

Another study has evaluated the VT characteristics and ablation outcomes in 20 patients with prior valve surgery including 6 patients with lone MVS and 2 patients with mitral and aortic valve surgery. ${ }^{10}$ Four patients had VT within 30 days of surgery, 2 of them were non-inducible, 1 was BBR VT and 1 was scarrelated reentry. On the other hand, $81 \%$ of patients with late-onset VT (1 to 16 years after surgery) had scar-related reentry VT. Out of the 8 patients with MVS, 6 patients had inducible VT at EP study where scar-related reentry was the mechanism of VT in 5 patients and automatic VT was found in one patient. In 3 out of the 6 MVS patients with inducible VT, the origin of arrhythmia involved the mitral valve annulus with RBB morphology. This is consistent with our findings where RBB was the dominant morphology. However, the origin of VA did not involve the mitral annulus in the majority of our cohort.

Although electrophysiological scar was a universal finding in our patients, it is difficult to identify whether perimitral scar is related to MVS or the primary valve pathology that led to MVS. However, the absence of mitral annulus disjunction in our cohort may suggest that the scar was related to MVS though was often not critical to the observed arrhythmias. ${ }^{26,27}$

\section{Left ventricular access}


Access to LV can generally be achieved through an anterograde transseptal or aortic retrograde approach. However, access to the LV could be challenging with previous heart surgery especially with the presence of mechanical valves. In our series, 17 patients underwent antegrade approach, 13 patients had retrograde aortic approach, 1 patient had a combined approach. Only 1 patient had a percutaneous epicardial approach following a failed ablation through antegrade-transseptal approach during the same procedure. As expected, all patients with a mechanical mitral valve underwent retrograde approach.

The presence of mechanical MV may slightly hinder ablation due to one less route of access to the LV which may reduce maneuverability and contact force. ${ }^{26,27}$ Oftentimes, a combined approach is required to maximize mapping and allow sufficient access to certain regions e.g. the posterior papillary muscle. ${ }^{28}$

While no patients in our cohort had inaccessible LV, in certain circumstances such as the presence of mechanical aortic and mitral valves, non-conventional techniques might be necessary to access LV. In such cases, options would include epicardial approach, ${ }^{29}$ transventricular septal puncture ${ }^{30}$ transcoronary venous approach,${ }^{31}$ transapical approach, ${ }^{32}$ or trans-right atrial to the LV approach. ${ }^{33}$

Percutaneous epicardial access can be challenging in patients with prior cardiac surgery. A previous study reported that prior cardiac surgery precludes LV access in most patients with only $20 \%$ success rate. ${ }^{34}$ Other reports demonstrated the feasibility of percutaneous epicardial approach in patients with prior cardiac surgery. ${ }^{35,36}$ In one patient, we used transcutaneous epicardial approach as endocardial mapping did not show an obvious exit of the VT. Epicardial access was very challenging in this patient due to adhesions associated with prior surgery and the presence of pectus excavatum deformity. However, this was overcome with a more anterior approach and a complete epicardial map was achieved with successful VT ablation.

\section{Safety and outcomes of ablation}

The presence of a mechanical mitral valve for VA ablation can pose unique operative risks. Strict attention with respect to periprocedural anticoagulation is required to minimize the risk of thromboembolism or valve thrombosis. In addition, meticulous care is required when mapping around the valve to avoid catheter entrapment. We typically avoid use of a multispline ${ }^{37}$ catheter in patients with mechanical valves due to the risk of entrapment and device shearing. In addition, use of ICE is strongly encouraged to help more accurately determine anatomical locality. In addition, fluoroscopic imaging in the right anterior oblique plane would help provide information regarding proximity to the mitral valve. In the rare and potential catastrophic event of catheter entrapment during mapping or ablation, management includes cautious catheter rotation/ manipulation in various planes to free up the catheter. Advancement of a support sheath up to the level of obstruction can be entertained. Furthermore, measures to prolong mitral valve opening such as rapid ventricular pacing and adenosine (which prolong diastole) can be attempted. ${ }^{38}$ If these measures fail, one can employ extraction techniques or ultimately consider surgical removal.

Thromboembolic events are uncommon, yet devastating complication of VT ablation. Ventricular ablation is associated with an inherent risk of thrombus formation with thrombus size directly proportional to ablative lesion size. ${ }^{39}$ Reported incidence of stroke/ TIA with VT ablation ranges from $0.8 \%$ to $1.8 \%{ }^{40,41}$ Studies have shown that the incidence of thromboembolic events with VT ablation is higher in patients with structural heart disease. ${ }^{42}$ In our study, no thromboembolic events or other procedure-related complications were reported. ICE was performed before and after the ablation did not detect any change of valve function or development of pericardial effusion. Ecktar et al reported a series of VT ablation in 20 patients including 8 patients with MVS. ${ }^{10}$ In their study, during a median follow-up of 2.1 years, $11(55 \%)$ patients remained free of spontaneous VT. Three patients had repeat ablation due to recurrent VT. In our study, the overall VA recurrence-free rate at 1-year was $13 / 18(72.2 \%)$. We also found that MVS patients with a history of CAD showed a trend of better VA recurrence-free survival compared with those without CAD history. (Figure 2B ) This could be related to the previously reported different nature of VT circuits between ICM and NICM. ${ }^{43,44}$ One observational study compared the characteristics and the outcomes of VT ablation between ICM and NICM patients has shown that complete success and 1-year VT-free survival were higher in the ICM group. ${ }^{44}$ The critical isthmus for VT was identified with endocardial entrainment in $62 \%$ of the ICM group 
and in $17 \%$ of the NICM group. The lower likelihood of identifying the isthmus in NICM might be related to a midmyocardial or epicardial location, which is a less amenable location for successful ablation especially in patients with previous cardiac surgery.

\section{Limitations}

We acknowledge several limitations, some of which are inherent to the retrospective analysis. First, the sample size included in the study was small. However, given the limited data in literature about this specific population, our sample size is one of the biggest for this category of patients. As this is a single-center analysis, results may not be generalizable. Also, our patients tend to be referred to us late after multiple failed therapeutic trials, which could affect the findings. We were unable to determine the cause of death in 2 patients. Therefore, we cannot exclude recurrent ventricular arrhythmia as the cause of death in these patients.

\section{Conclusions}

Ventricular arrhythmias in patients with prior MVS can be treated safely and effectively with catheter ablation. Though all patients in our study had prior MVS, clinical VA did not originate from the mitral valve area in the majority of patients.

\section{Data availability}

The data underlying this article will be shared on reasonable request to the corresponding author.

\section{References}

1. Kligfield P, Hochreiter C, Niles N, Devereux RB, Borer JS. Relation of sudden death in pure mitral regurgitation, with and without mitral valve prolapse, to repetitive ventricular arrhythmias and right and left ventricular ejection fractions. The American Journal of Cardiology. 1987;60(4):397-399.

2. Delahaye JP, Gare JP, Viguier E, Delahaye F, De Gevigney G, Milon H. Natural history of severe mitral regurgitation. European heart journal. 1991;12 Suppl B:5-9.

3. Hochreiter C, Niles N, Devereux RB, Kligfield P, Borer JS. Mitral regurgitation: relationship of noninvasive descriptors of right and left ventricular performance to clinical and hemodynamic findings and to prognosis in medically and surgically treated patients. Circulation. 1986;73(5):900-912.

4. Duren DR, Becker AE, Dunning AJ. Long-term follow-up of idiopathic mitral valve prolapse in 300 patients: a prospective study. Journal of the American College of Cardiology. 1988;11(1):42-47.

5. Boudoulas H, Schaal SF, Stang JM, Fontana ME, Kolibash AJ, Wooley CF. Mitral valve prolapse: cardiac arrest with long-term survival.International journal of cardiology. 1990;26(1):37-44.

6. Blackstone EH, Kirklin JW. Death and other time-related events after valve replacement. Circulation. 1985;72(4):753-767.

7. Narasimhan C, Jazayeri MR, Sra J, et al. Ventricular Tachycardia in Valvular Heart Disease. Circulation. 1997;96(12):4307-4313.

8. Naksuk N, Syed FF, Krittanawong C, et al. The effect of mitral valve surgery on ventricular arrhythmia in patients with bileaflet mitral valve prolapse. Indian Pacing Electrophysiol J.2016;16(6):187-191.

9. Vaidya VR, DeSimone CV, Damle N, et al. Reduction in malignant ventricular arrhythmia and appropriate shocks following surgical correction of bileaflet mitral valve prolapse. J Interv Card Electrophysiol. 2016;46(2):137-143.

10. Eckart RE, Hruczkowski TW, Tedrow UB, Koplan BA, Epstein LM, Stevenson WG. Sustained ventricular tachycardia associated with corrective valve surgery. Circulation. 2007;116(18):2005-2011. 
11. Narasimhan C, Jazayeri MR, Sra J, et al. Ventricular tachycardia in valvular heart disease: facilitation of sustained bundle-branch reentry by valve surgery. Circulation. 1997;96(12):4307-4313.

12. Fedgchin B, Pavri BB, Greenspon AJ, Ho RT. Unique self-perpetuating cycle of atrioventricular block and phase IV bundle branch block in a patient with bundle branch reentrant tachycardia. Heart rhythm.2004;1(4):493-496.

13. Lewalter T, Jung W, Preusse CJ, et al. Radiofrequency catheter ablation of an incessant ventricular tachycardia following valve surgery. Pacing and clinical electrophysiology : PACE.2002;25(1):105-108.

14. Steinberg JS, Gaur A, Sciacca R, Tan E. New-onset sustained ventricular tachycardia after cardiac surgery. Circulation.1999;99(7):903-908.

15. Isa Param R, Perez-Castellano N, Villacastin J, Moreno J, Ruiz E, Solis J. [Optimized transseptal approach for left ventricular tachycardia ablation in a patient with a mechanical prosthetic aortic valve]. Revista espanola de cardiologia. 2005;58(6):756-758.

16. Liang JJ, Castro SA, Muser D, et al. Electrophysiologic Substrate, Safety, Procedural Approaches, and Outcomes of Catheter Ablation for Ventricular Tachycardia in Patients After Aortic Valve Replacement.JACC Clinical electrophysiology. 2019;5(1):28-38.

17. Kella DK, Sheldon SH, Noheria A, et al. Defining the substrate for ventricular tachycardia ablation: The impact of rhythm at the time of mapping. Indian Pacing Electrophysiol J. 2020;20(4):147-153.

18. Latchamsetty R, Yokokawa M, Morady F, et al. Multicenter Outcomes for Catheter Ablation of Idiopathic Premature Ventricular Complexes.JACC Clinical electrophysiology. 2015;1(3):116-123.

19. Lukac P, Hjortdal VE, Pedersen AK, Mortensen PT, Jensen HK, Hansen PS. Atrial incision affects the incidence of atrial tachycardia after mitral valve surgery. The Annals of thoracic surgery.2006;81(2):509-513.

20. Mountantonakis S, Frankel DS, Hutchinson MD, et al. Feasibility of catheter ablation of mitral annular flutter in patients with prior mitral valve surgery. Heart Rhythm. 2011;8(6):809-814.

21. Viles-Gonzalez JF, Enriquez AD, Castillo JG, et al. Incidence, predictors, and evolution of conduction disorders and atrial arrhythmias after contemporary mitral valve repair. Cardiol J.2014;21(5):569-575.

22. Yeung-Lai-Wah JA, Qi A, McNeill E, et al. New-onset sustained ventricular tachycardia and fibrillation early after cardiac operations. The Annals of thoracic surgery. 2004;77(6):2083-2088.

23. Smith R, Grossman W, Johnson L, Segal H, Collins J, Dalen J. Arrhythmias following cardiac valve replacement. Circulation.1972;45(5):1018-1023.

24. Ware JA, Magro SA, Luck JC, et al. Conduction system abnormalities in symptomatic mitral valve prolapse: An electrophysiologic analysis of 60 patients. The American Journal of Cardiology.1984;53(8):10751078 .

25. Marchenese K, Schenk E. Atrioventricular conduction system lesion following cardiac valve replacement. Circulation. 1972;45(Suppl. II):II 188.

26. Dejgaard LA, Skjolsvik ET, Lie OH, et al. The Mitral Annulus Disjunction Arrhythmic Syndrome. Journal of the American College of Cardiology. 2018;72(14):1600-1609.

27. Perazzolo Marra M, Basso C, De Lazzari M, et al. Morphofunctional Abnormalities of Mitral Annulus and Arrhythmic Mitral Valve Prolapse.Circ Cardiovasc Imaging. 2016;9(8):e005030-e005030.

28. Mizuno H, Vergara P, Maccabelli G, et al. Contact force monitoring for cardiac mapping in patients with ventricular tachycardia. Journal of cardiovascular electrophysiology. 2013;24(5):519-524.

29. Khalil F, Siontis K, Bagameri G, Killu AM. Hybrid Catheter-Based and Surgical Techniques for Ablation of Ventricular Arrhythmias.Arrhythmia \&3 electrophysiology review. 2020;9(2):97-103. 
30. Santangeli P, Shaw GC, Marchlinski FE. Radiofrequency Wire Facilitated Interventricular Septal Access for Catheter Ablation of Ventricular Tachycardia in a Patient With Aortic and Mitral Mechanical Valves. Circulation Arrhythmia and electrophysiology. 2017;10(1).

31. Najjar J, Bortone A, Boveda S, Albenque J-P. Radiofrequency ablation of an epicardial ventricular tachycardia through the great cardiac vein in a patient with mitro-aortic mechanical prostheses. EP Europace. 2007;9(11):1069-1072.

32. Vurgun VK, Altin AT, Kilickap M, Candemir B, Akyurek O. Percutaneous transapical approach and transcatheter closure for ventricular tachycardia ablation. Pacing and clinical electrophysiology : PACE. 2018;41(3):334-337.

33. Santangeli P, Hyman MC, Muser D, Callans DJ, Shivkumar K, Marchlinski FE. Outcomes of Percutaneous Trans-Right Atrial Access to the Left Ventricle for Catheter Ablation of Ventricular Tachycardia in Patients With Mechanical Aortic and Mitral Valves. JAMA Cardiology. 2020.

34. Roberts-Thomson KC, Seiler J, Steven D, et al. Percutaneous access of the epicardial space for mapping ventricular and supraventricular arrhythmias in patients with and without prior cardiac surgery. Journal of cardiovascular electrophysiology. 2010;21(4):406-411.

35. Soejima K, Couper G, Cooper JM, Sapp JL, Epstein LM, Stevenson WG. Subxiphoid Surgical Approach for Epicardial Catheter-Based Mapping and Ablation in Patients With Prior Cardiac Surgery or Difficult Pericardial Access. Circulation. 2004;110(10):1197-1201.

36. Killu AM, Ebrille E, Asirvatham SJ, et al. Percutaneous Epicardial Access for Mapping and Ablation Is Feasible in Patients With Prior Cardiac Surgery, Including Coronary Bypass Surgery. Circulation: Arrhythmia and Electrophysiology. 2015;8(1):94-101.

37. Desimone CV, Hu T, Ebrille E, et al. Catheter ablation related mitral valve injury: the importance of early recognition and rescue mitral valve repair. J Cardiovasc Electrophysiol.2014;25(9):971-975.

38. Sheldon SH, Good E. PentaRay entrapment in a mechanical mitral valve during catheter ablation of atrial fibrillation. HeartRhythm Case Rep. 2015;2(2):200-201.

39. Khairy P, Chauvet P, Lehmann J, et al. Lower Incidence of Thrombus Formation With Cryoenergy Versus Radiofrequency Catheter Ablation.Circulation. 2003;107(15):2045-2050.

40. Kuck KH, Schaumann A, Eckardt L, et al. Catheter ablation of stable ventricular tachycardia before defibrillator implantation in patients with coronary heart disease (VTACH): a multicentre randomised controlled trial. Lancet. 2010;375(9708):31-40.

41. Bohnen M, Stevenson WG, Tedrow UB, et al. Incidence and predictors of major complications from contemporary catheter ablation to treat cardiac arrhythmias. Heart Rhythm. 2011;8(11):1661-1666.

42. Peichl P, Wichterle D, Pavlu L, Cihak R, Aldhoon B, Kautzner J. Complications of Catheter Ablation of Ventricular Tachycardia.Circulation: Arrhythmia and Electrophysiology. 2014;7(4):684-690.

43. Chung F-P, Lin C-Y, Lin Y-J, et al. Ventricular arrhythmias in nonischemic cardiomyopathy. $J$ Arrhythm. 2018;34(4):336-346.

44. Shirai Y, Liang JJ, Santangeli P, et al. Comparison of the Ventricular Tachycardia Circuit Between Patients With Ischemic and Nonischemic Cardiomyopathies. Circulation: Arrhythmia and Electrophysiology. 2019;12(7):e007249.

\section{Figure legends}

Figure 1: Overall (a) and arrhythmia recurrence-free (b) survival.

Figure 2: Overall (a) and arrhythmia recurrence-free (b) survival according to presence or absence of coronary artery disease. 
Table 1. Baseline characteristics

Table 2. General characteristics of VA and ablation

Table 3. Characteristics of VT in patients with mitral valve surgery

Table 4. Characteristics of PVC in patients with mitral valve surgery

\section{Hosted file}

Table 1.docx available at https://authorea.com/users/423297/articles/528792-characteristicsand-outcomes-of-ventricular-tachycardia-and-premature-ventricular-contractions-ablationin-patients-with-prior-mitral-valve-surgery

\section{Hosted file}

Table 2.docx available at https://authorea.com/users/423297/articles/528792-characteristicsand-outcomes-of-ventricular-tachycardia-and-premature-ventricular-contractions-ablationin-patients-with-prior-mitral-valve-surgery

\section{Hosted file}

Table 3.docx available at https://authorea.com/users/423297/articles/528792-characteristicsand-outcomes-of-ventricular-tachycardia-and-premature-ventricular-contractions-ablationin-patients-with-prior-mitral-valve-surgery

\section{Hosted file}

Table 4.docx available at https://authorea.com/users/423297/articles/528792-characteristicsand-outcomes-of-ventricular-tachycardia-and-premature-ventricular-contractions-ablationin-patients-with-prior-mitral-valve-surgery 
Figure 1

Figure 1A. Survival

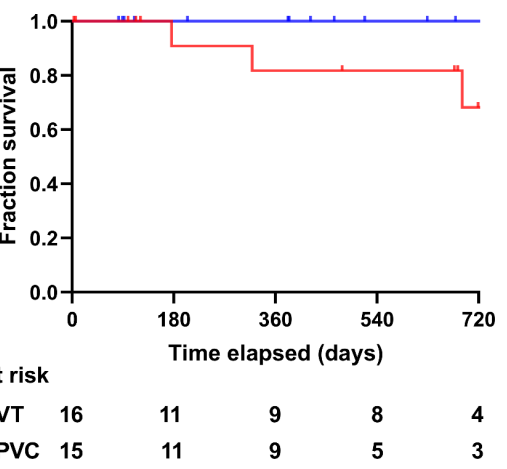

Figure 1B. Recurrence-free survival

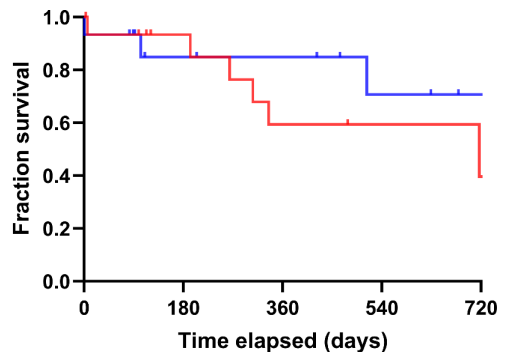

No. at risk

$\begin{array}{rlllll}-\mathrm{VT} & 16 & 11 & 7 & 6 & 4\end{array}$

$\begin{array}{rrrrr}- \text { PVC } & 15 & 9 & 8 & 5\end{array}$ 
Figure 2

Figure 2A. Survival (CAD vs. no CAD) following VT ablation

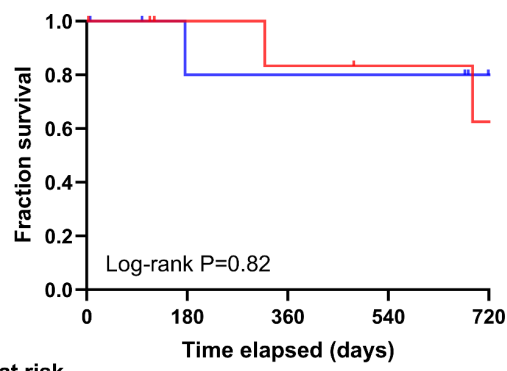

No. at risk

$\begin{array}{lrllll}-{ }_{-} \text {CAD } & 9 & 6 & 5 & 4 & 3 \\ - \text { No CAD } 7 & 5 & 2 & 2 & 1\end{array}$

Figure 2B. Recurrence-free survival (CAD vs. no CAD) following VT ablation

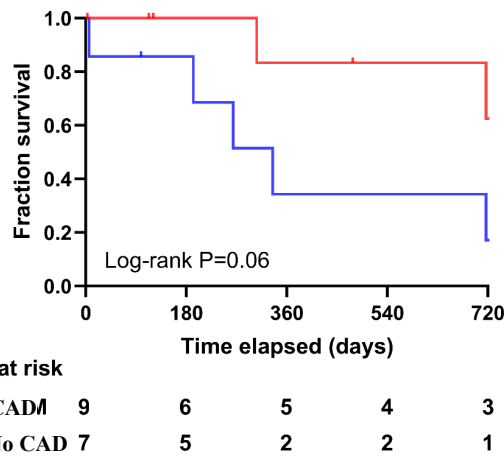

